\title{
Medical ultrasound image segmentation by modified local histogram range image method
}

\author{
Ali Kermani, Ahmad Ayatollahi, Ahmad Mirzaei, Mohammad Barekatain
}

School of Electrical Engineering, Iran University of Science and Technology, Tehran, Iran.

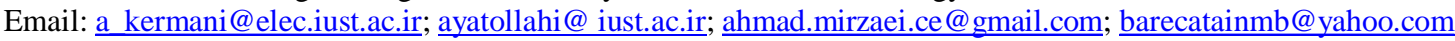

Received 8 September 2010; revised 8 October 2010; accepted 13 October 2010.

\begin{abstract}
Fast and satisfied medical ultrasound segmentation is known to be difficult due to speckle noises and other artificial effects. Since speckle noise is formed from random signals which are emitted by an ultrasound system, we can't encounter the same way as other image noises. Lack of information in ultrasound images is another problem. Thus, segmentation results may not be accurate enough by means of customary image segmentation methods. Those methods that can specify undesirable effects and segment them by eliminating artificial effects, should be chosen. It seems to be a complicated work with high computational load. The current study presents a different approach to ultrasound image segmentation that relies mainly on local evaluation, named as local histogram range image method which is modified by means of discrete wavelet transform. Thus, a significant decrease in computational load is then achieved. The results show that it is possible for tissues to be segmented correctly.
\end{abstract}

Keywords: Segmentation; Local Histogram; Ultrasound Image; Morphological Image Processing; Discrete Wavelet Transform

\section{INTRODUCTION}

Fast and reliable ultrasound image segmentation is a complicated process with particular difficulties. Because of the presence of speckle in these images and their poor contrast, using common methods for segmentation is not possible. General approaches to segmentation can be grouped into three categories: 1) pixel-based, 2) continuity, and 3) edge based methods. Many segmentation methods that are presented, include clustering, watershed, active contours, and those methods that are based on statistical models [1-4]. Pixel-based methods are the easiest to implement, since they apply to one element while, they are particularly susceptible to noise. Continuity-based and edge-based methods encounter the segmentation problem from opposing sides: continuitybased methods search for similarities while edge-based methods search for differences [5].

On the other hand, almost these segmentation methods consider the entire image as a unit, which contains a large area of gray value, and uses spatial or frequency for segmenting; however, it can't recognize speckle noises accurately. Local evaluation methods can be a solution for ultrasound images, since speckle pixels will be recognized precisely. In [6], local estimation of Rayleigh parameter was proposed to identify different tissues. However, its complexity and computational load are high. In [7], researchers defined "local histogram range image" (LHRI) based on histogram distribution which reduced statistic complexity. They applied a classification method to recognize edge or border of organ while, speckle noises remained unchanged. LHRI was proposed for segmentation in [8]; although, its computational load was too high.

In this study, the combined edge-based and continuitybased method are presented by modified LHRI. Discrete wavelet transform (DWT) is applied to reduce dimension of input images of LHRI algorithm; whereas, the image energy compresses in few number of wavelet's coefficients. Hence, we expect to have proper segmented images and to decrease computational load by means of reducing input image size.

We will introduce the LHRI method for an ultrasound image in Part 2. Section 3 describes DWT. Combination of LHRI method with DWT is introduced in Section 4. In Section 5 the simulation results are presented.

\section{LOCAL HISTOGRAM RANGE IMAGE FOR SEGMENTATION}

\subsection{Definition}

The recognition of ultrasound signal characteristics is the primary requirement. Speckle has a random nature as 
it is formed from signals which are produced by scatterers in the medium under observation. This analysis of speckles is one of the most important studies. In addition, today's advanced scanners apply a log-compression to the envelope data to enhance the lower signal values. Considering that log-compression is a nonlinear operation, statistics of envelope signal are different before and after this compression.

Because of different scatterer's distribution and density in different tissues, researchers have studied several probability density models of ultrasound envelope signals. We divide image areas into two general groups to evaluate an ultrasound image: 1-fully developed speckle (a large number of randomly located scatterers with small scatterer spacing were compared to the wavelength of ultrasound), 2-areas that have low scatterers. Several distributions are presented for each mentioned area that we only introduce two usual distributions. Statistics demonstrate Rayleigh model in fully developed speckle areas [9]. In the second area that the number of scatterers is low and its spatial locations are dependent variables, Nakagami distribution is the most flexible model among the others [10]. Of course, these distributions are due to previous logarithmic compression step. However, reference [7] has shown that each distribution can be achieved after applying logarithmic compression. For the first area (fully developed speckle), Raleigh distribution convert into double exponential distribution that its mean and variance are obtained as follows:

$$
\begin{gathered}
\text { Mean }_{\text {Log-Rayleigh }}=\frac{n_{1} \ln (2)}{2}+n_{1} \ln (\sigma)-\frac{\gamma n_{1}}{2}+n_{2}, \\
V A R_{\text {Log-Rayleigh }}=E\left[(y-\bar{y})^{2}\right]=\frac{\pi^{2} n_{1}^{2}}{24}
\end{gathered}
$$

where $\gamma=0.5772$ is Euler constant, $n_{1}$ is image dynamic range, and $n_{2}$ is gain setting (ratio of minimum to maximum output signal). It can be concluded that the mean depends on three factors: initial Raleigh variance, $n_{1}$ and $n_{2}$, while the variance only depends on dynamic range $n_{1}$.

Also, log-Nakagami [9] was derived for latter area that its variance is as follows:

$$
\operatorname{VAR}_{\text {Log-Nakagami }}=E\left[(y-\bar{y})^{2}\right]=\frac{n_{1}^{2}}{4} \xi(m),
$$

where $\xi$ is defined as follow:

$$
\xi(m)=\frac{1}{m^{2}}+\frac{1}{(m+1)^{2}}+\ldots,
$$

Log-Nakagami distribution tends to Rician in the case of $\mathrm{m}=1.5$, which leads to the variance below:

$$
V_{\text {Log-Rice }}=E\left[(y-\bar{y})^{2}\right]=\frac{\pi^{2} n_{1}^{2}}{42.23} .
$$

Since both distributions depend on one parameter $\left(n_{1}\right)$, their comparison shows that the variance of logRayleigh exceeds the variance of log-Nakagami. Hence, variance of log-Rayleigh is more than log Nakagami's variance.

After introducing the distribution, now it's the time to find out the relationship between borders and mentioned distributions. The borders of ultrasound images are totally divided into two groups: 1) the edge between two fully developed speckle areas, and 2) the Edge between speckle area and specular scattered areas. The experiments on ultrasound images show that although histograms of fully developed speckle areas have similar structure; however, their mean values could be different for these two different organs. Also, equal variances and different means are concluded from double exponential equations. In addition, local histogram range in borders between two fully developed speckle areas is more than the range of local histogram in fully developed areas. We can see from (5) that the variance of Rician distribution after log compression will be a constant, and its value is smaller than the one in Rayleigh distribution. Therefore, valid range of the histogram will not be very large, while its mean is larger than double exponential mean. Local histogram between two specular scatterer and speckle has the largest range among other areas.

We can recognize edges, and segment image by means of proper function via prior analysis and relation between distributions and valid local histogram range, without high computation load of statistic calculations. Therefore, we use local histogram range image definition [7]: at first we should choose proper size for moving window that suits our approach. The experiment on several ultrasound images shows that the best window size for segmentation is $4 \times 4$ to $15 \times 15$. This window moves around original image and an $N \times M$ matrix is obtained as below:

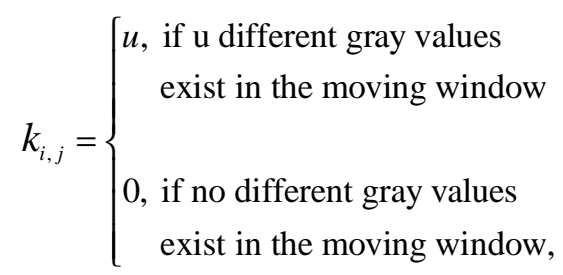

where $k_{i, j}$ is the pixel value of LHRI at the position a $(\mathrm{i}$, j).

\subsection{Classifier Function}

Here, a function is described that separates different image areas after computation of the LHRI matrix. In [7], 
authors have used a linear adaptive function to enhance edges; we modified it for segmentation. Our purpose is clustering pixels in two levels, foreground and background. The mean of whole image is a general margin for separating two classes, but it is a static parameter; therefore, it doesn't work properly for all ultrasound images. We need dynamic parameter to classify pixels truly. For this reason, we define classifier function below with dynamic parameter $k_{i, j}$ obtained from LHRI matrix:

$$
f_{i, j}=g_{i, j}+\alpha k_{i, j}\left(g_{i, j}-\bar{g}\right)
$$

where $f_{i, j}$ is the enhanced value, $g_{i, j}$ is the old one, $\bar{g}$ is the mean of whole image, and $\alpha$ is the coefficient value. Distance of each pixel's gray value from mean is computed at first in this equation. This distance can be positive or negative. Output pixel would be brighter if this value is positive, and darker if it is negative. The proportion of this change depends on $k_{i, j}$. It can be explained as local adaptive classifying parameter, whereas more $k_{i, j}$ causes more margin parameter near the whole image mean.

Figure 1 shows this function for 8 bit gray level inputs and several k ( 0 to 9 ) and $\bar{g}=127$. It can be seen that the defined function classifies and ranks gray level values of pixels in terms of LHRI pixel value and whole image mean.

Another problem with this approach is the $\alpha$ value. Implementation of this algorithm indicates that proper results would be attained by coefficient values between 0.1 to 1 which depend on gray value range of original image.

\subsection{Morphological Image Processing}

Since LHRI algorithm leads to the existence of small holes in the obtained matrix, it can't classify tissues properly. For this reason, we apply morphological image processing to achieve perfect and smooth tissues. Consequently, dilation and erosion are selected. At first, we apply dilation to eliminate holes and fill the contour gaps [12]. The dilation is defined as:

$$
A \oplus B=\left\{z \mid(B)_{z} \cap A \neq \varphi\right\}
$$

where... is a median filtered image, $B$ is a circle or a square structuring element and denotes reflection of $\mathrm{B}$. Erosion is applied to eliminate the effects caused by dilation in the tissue size. It is defined as:

$$
A \Theta B=\left\{z \mid(B)_{z} \subseteq A\right\}
$$

The key problem with this explanation is the size of structuring element, because small structuring doesn't fill holes correctly. Furthermore, large size of them makes artificial edges. Experiments show that $3 \times 3$ element could fill holes properly.

\section{DISCRETE WAVELET TRANSFORM}

The concept of "wavelet" was first introduced in 1984

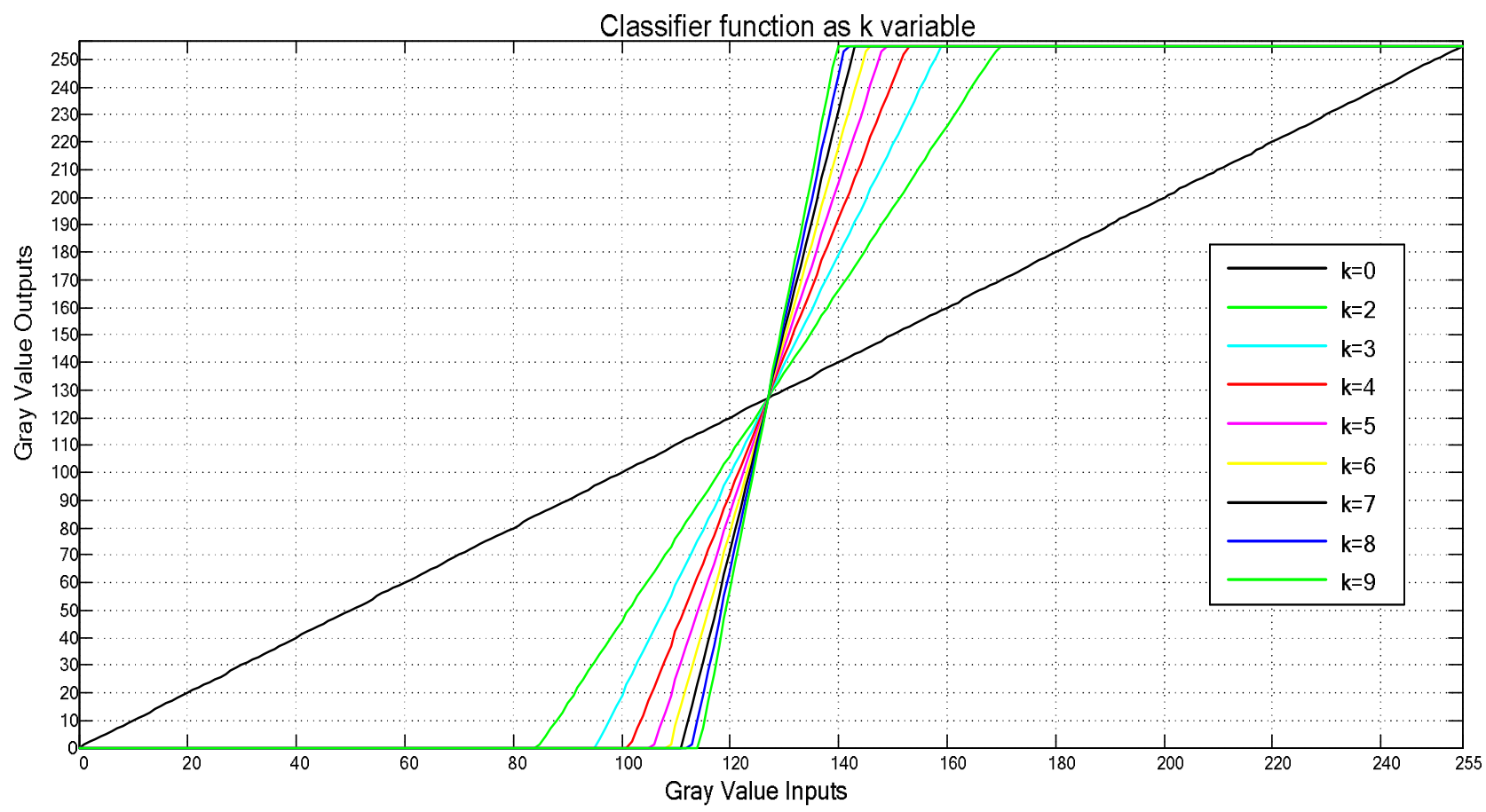

Figure 1. Classifier function for $\mathrm{k}=\{0,2,3, \ldots, 9\}$. 
by A. Grossmann and J. Morlet [13]. By wavelet transform (WT), each signal included in $L^{2}(R)$ is presented as weighted summation of wavelet basis functions. In WT, the basis functions are obtained from a single function named "wavelet basis functions" by time translation (b) and dilation (a). Eq.10 presents wavelet function:

$$
\psi_{b, a}(x)=\frac{1}{\sqrt{a}} \times \psi\left(\frac{x-b}{a}\right)
$$

If $a$, and $b$, in continuous wavelet transform, are used in binary form, the DWT is yielded [14]:

$$
a=2^{m}, b=2^{m} \times n
$$

Thus, DWT is defined as:

$$
W_{f}\left(2^{m} n, 2^{m}\right)=2^{-\frac{m}{2}} \int_{-\infty}^{+\infty} \psi\left(2^{-m} x-n\right) f(x) d x,
$$

Inverse discrete wavelet transform (IDWT) is obtained by following equation:

$$
f(x)=\sum_{m=-\infty}^{+\infty} \sum_{n=-\infty}^{+\infty} W_{f}\left(2^{m} n, 2^{m}\right) \Psi_{m n}(x)
$$

Each wavelet basis function has nonzero value in special frequency interval (band). Therefore, it concludes signal information in the special frequency interval. Also, basis functions are selected in a way that they are orthogonal. This means that there are no overlaps between the special frequency intervals of various basis functions [14]. This special frequency interval of wavelet basis is introduced as follows:

$$
W_{m} \equiv \operatorname{span}\left\{\Psi\left(2^{-m} x-n\right)\right\} \quad m \in Z .
$$

Another set of functions named scaling function, is introduced in a way that its special frequency band comes from union the special frequency interval of wavelet basis. If its special frequency band of scaling function is defined as $V_{m}$, we have [14]:

$$
V_{m}=\bigcup_{i=-\infty}^{m-1} W_{i}, \mathrm{~V}_{-\infty}=\varphi, \mathrm{V}_{+\infty}=L^{2}(R)
$$

Scaling functions that are orthogonal basis for $V_{m}$, are obtained thorough time translation and dilation of special function as follows:

$$
\varphi_{n, m}(x)=2^{-\frac{m}{2}} \varphi\left(2^{-m} x-n\right) \mathrm{n} \in \mathrm{Z}
$$

Therefore, for survey of the signal in all frequency bands, DWT should be calculated for $m \in[a,+\infty)$ along with signal decomposition with $\varphi_{(a-1) n}$ [15]. In such a way signal decomposition is as follows:

$$
f(x)=\sum_{k} d_{j_{0} k} \varphi\left(2^{j_{0}} x-k\right)+\sum_{j=j_{0}}^{+\infty} \sum_{k} c_{j k} 2^{j / 2} \psi\left(2^{j} x-k\right)
$$

where $c_{j k}$ and $d_{j_{0} k}$ are approximations and details coefficients of WT respectively, and they are computed by:

$$
\begin{aligned}
d_{j_{0} k} & =\int_{-\infty}^{+\infty} f(x) \varphi_{j_{0} k}(x) d x \\
c_{j k} & =\int_{-\infty}^{+\infty} f(x) \Psi_{j k}(x) d x
\end{aligned}
$$

These coefficients for discrete signal are calculated by [16]:

$$
\begin{aligned}
c_{j n} & =\sum_{k} h(k-2 n) c_{j+1, k} \\
d_{j n} & =\sum_{k} g(k-2 n) c_{j+1, k}
\end{aligned}
$$

where $h(k)$ low pass filter coefficient and $g(k)$ is high pass filter coefficient.

We need to generalize mentioned subjects into two dimensions under to apply DWT on images. Four fundamental functions must be used for two dimensional DWT, which elicit low frequency information and high frequency information in three directions ' $x$ ', ' $y$ ' and diagonal. These functions are assumed to be separable for simplicity. Therefore, we have from one dimensional wavelet and scaling functions [17]:

$$
\begin{aligned}
& \varphi(x, y)=\varphi(x) \varphi(y) \\
& \psi^{V}(x, y)=\varphi(x) \psi(y) \\
& \psi^{h}(x, y)=\psi(x) \varphi(y) \\
& \psi^{d}(x, y)=\psi(x) \psi(y)
\end{aligned}
$$

where these functions compute four types of coefficients: approximation, vertical, horizontal and diagonal coefficients. Two dimensional DWT computations is converted to double calculation of one dimensional DWT by using separable mentioned functions.

Input image is divided into four images which consists of approximation image, vertical, horizontal and diagonal edges for each decomposition level in DWT. Approximation part will be a shrank image that contains a large amount of primary image energy. So we can apply some image processing algorithms (such as segmentation) on approximation coefficients to decrease computation load.

\section{COMBINATION OF LHRI METHOD WITH DWT}

As mentioned in previous section, for decreasing execution time of algorithm, we can calculate approximation coefficients from DWT, which have a large amount of image energy, and then use them in LHRI algorithm.

It should be noted that using DWT and its inverse in proper portions of algorithm is extremely important. In 
general we can do it in two forms. We apply them and choose the best one by comparing them.

In the first method, we determine DWT of original image, and then approximated wavelet coefficients are used as LHRI input. We calculate IDWT before applying local histogram range image (LHRI) on primary image. Subsequently, the obtained matrix is defined as LHRI matrix in next algorithm processes.

The difference between the second method and former is in using LHRI matrix coefficients on approximation coefficients of prior image, which is obtained with DWT. We finally use inverse transform for conclusion, to make similar output size with input image. We applied two introduced methods on almost fifty ultrasound images, and came to the conclusion that the former method segments images with more details. Figure 2 shows block diagram of proposed combined method. Step by Step implementation of this algorithm on an example image is demonstrated in Figure 3.

More specifically, if we apply DWT for more than one, the execution time of algorithm decreases considerably, and algorithm accuracy is reduced. Therefore, there is a trade-off between them. The experimental results show that two levels of decomposition have the best efficiency.

Input image size become quarter for adding each decomposition level in DWT, whereas wavelet computation time is low. Therefore, total computation time is reduced in similar ratio approximately. As a result, the execute time decreases in $1 / 16$ ratio for applying two decomposition levels.

\section{SIMULATION RESULTS}

We applied our algorithm on two images of carotid and breast lesion to evaluate the results. At first, we get DWT from input images. We should choose optimum filter which used in this transform. As [18] proved, we choose biorthogonal filter. Figure 4(a) shows original ultrasound breast lesion. Figure 4(b) illustrates segmented image by LHRI alone. As shown in Figure 4(c),

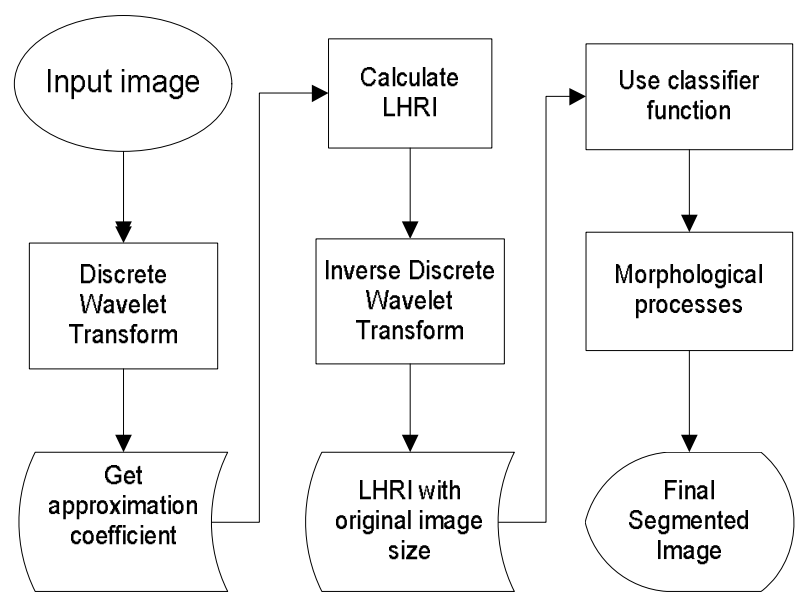

Figure 2. Block diagram of combined method.

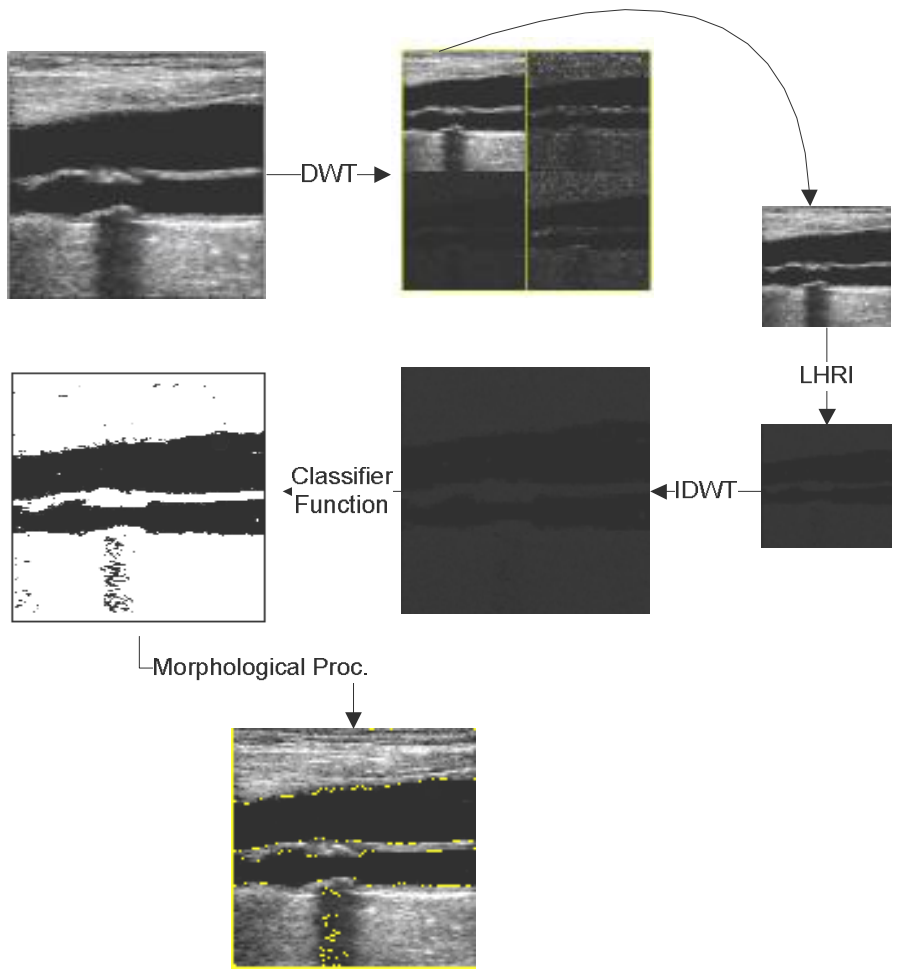

Figure 3. Step by step implementation of main algorithm. 


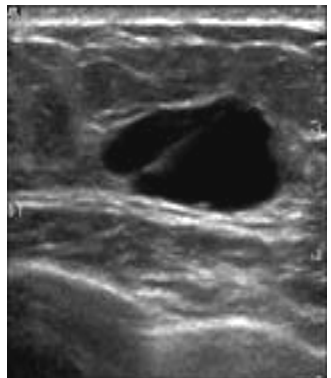

(a)

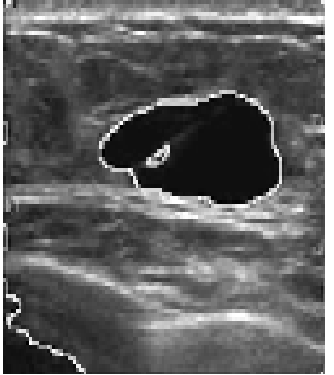

(b)

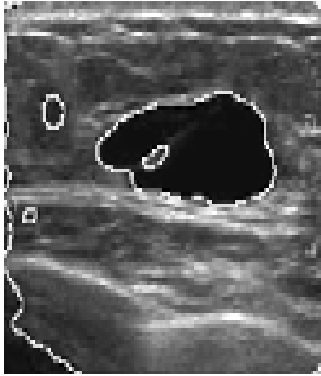

(c)

Figure 4. Segmentation of the ultrasound breast lesion image. (a) original image; (b) LHRI method result; (c) the method used in this study.

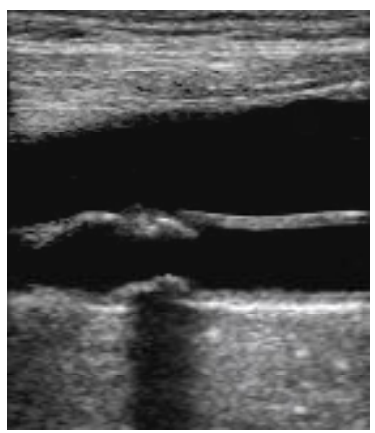

(a)

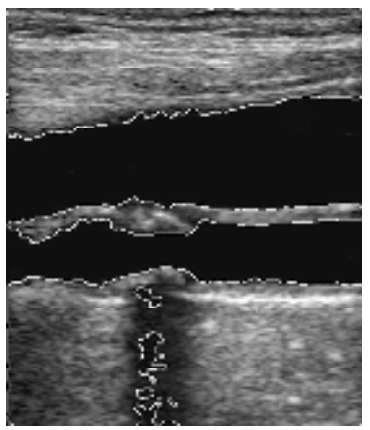

(b)

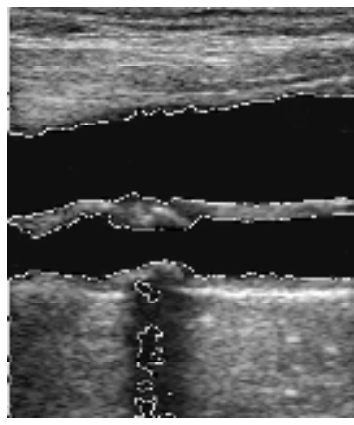

(c)

Figure 5. Segmentation of the ultrasound carotid image. (a) original image; (b)LHRI method result; (c) the method used in this study.

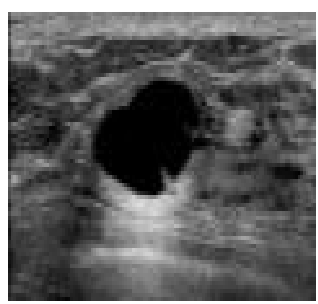

(a)

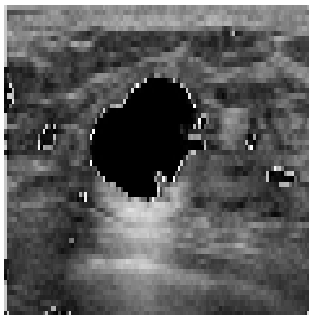

(d)

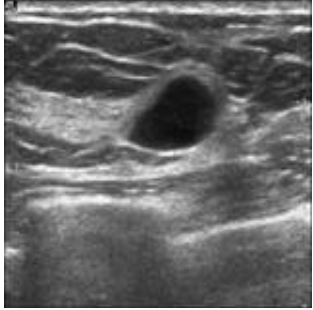

(b)

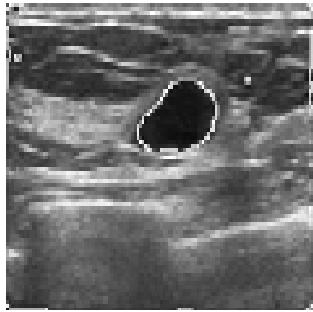

(e)

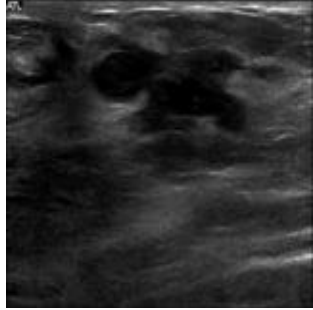

(c)

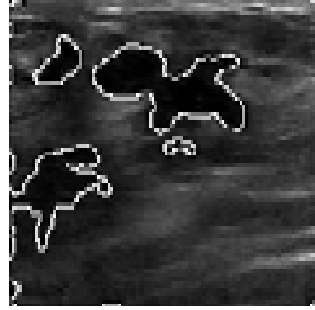

(f)

Figure 6. Breast lesion ultrasound image segmentation, (a, c, and e) original images, (b, d, and f) segmented results respectively.

segmentation result by LHRI \& wavelet method is similar to the former, whereas execution time is reduced considerably. We can prove these results for Figure 5, too. Table 1 shows the amount of simulation time for two algorithm implementations with $1.86 \mathrm{GHz}$ CPU by Matlab software for Figure 4. These times are illustrated for Figure 5 in Table 2. Figure 6 illustrates three breast lesions that are segmented by proposed method. 
Table 1. Execution time of simulation for Breast lesion.

\begin{tabular}{ccccc}
\hline & LHRI & DWT & IWT & $\begin{array}{c}\text { Total } \\
\text { Algorithm }\end{array}$ \\
\hline $\begin{array}{c}\text { LHRI } \\
\text { Algorithm }\end{array}$ & $1.054 \mathrm{sec}$ & - & - & $1.054 \mathrm{sec}$ \\
$\begin{array}{c}\text { LHRI }+ \\
\text { Wavelet }\end{array}$ & $0.127 \mathrm{sec}$ & $0.156 \mathrm{sec}$ & $0.016 \mathrm{sec}$ & $0.297 \mathrm{sec}$ \\
$\begin{array}{c}\text { Decrement } \\
\text { percent }\end{array}$ & $87.95 \%$ & - & - & $71.82 \%$ \\
\hline
\end{tabular}

Table 2. Execution time of simulation for carotid.

\begin{tabular}{ccccc}
\hline & LHRI & DWT & IWT & $\begin{array}{c}\text { Total } \\
\text { Algorithm }\end{array}$ \\
\hline $\begin{array}{c}\text { LHRI } \\
\text { Algorithm }\end{array}$ & $6.227 \mathrm{sec}$ & - & - & $6.227 \mathrm{sec}$ \\
$\begin{array}{c}\text { LHRI + } \\
\text { Wavelet }\end{array}$ & $0.549 \mathrm{sec}$ & $0.235 \mathrm{sec}$ & $0.078 \mathrm{sec}$ & $0.862 \mathrm{sec}$ \\
$\begin{array}{c}\text { Decrement } \\
\text { percent }\end{array}$ & $91.18 \%$ & - & - & $86.15 \%$ \\
\hline
\end{tabular}

We can see that the modified method by Wavelet segmented images with details and reduced implementation time about $90 \%$ of primary algorithm.

\section{CONCLUSIONS}

LHRI method segments envelope ultrasound images effectively. However, also its computation load is practically high. We show that DWT can decrease execution time without any change in segmentation result. Consequently, the required memory for algorithm implementation, is reduced. To obtain more accurate results while decreasing the computation load, we will use the modified wavelet and more advanced morphological proc

\section{REFERENCES}

[1] Chan, T. and Vese, L. (2001) Active contours without edges. IEEE Transaction on Image Processing, 10, 266272.

[2] Grau, V., Mewes, A.U.J., Alcaniz, M., Kikinis, R. and Warfield, S.K. (2004) Improved watershed transform for medical image segmentation using prior information IEEE Transactions on Medical Imaging, 23(4), 447-458.

[3] Wu, J. and Chung, A.C.S. (2007) “A segmentation model using compound Markov random fields based on a boundary model," IEEE Transaction on Image Processing, 16, 241-252.
[4] Makrogiannis, S., Economou, G., Fotopoulos, S. and Bourbakis, N.G. (2005) Segmentation of colour images using multiscale clustering and graph theoretic region synthesis. IEEE Transaction on System Man and Cybernetics, 35(2), 224-238.

[5] Semmlow, J.L. (2004) Biosignal and biomedical image processing. CRC Press, Boca Raton.

[6] Aja-Fern'andez, S., Martin-Fern'andez, M. and Alberola-L'opez, C. (2007) Tissue identification in ultrasound images using Rayleigh local parameter estimation. Proceedings of Bioinformatics and Bioengineering, Boston

[7] Wang, B. and Liu, D.C. (2008) A novel edge enhancement method for ultrasound imaging. The 2 nd International Conference on Bioinformatics and Biomedical Engineering, 3, 645-649.

[8] kermani, A., Ayatollahi, A. and Talebi, M. (2010) Segmentation medical ultrasound image based on Local histogram range image. The 3rd International Conference on BioMedical Engineering and Informatics, in Press.

[9] Shankar, P.M. (2000) A general statistical model for ultrasonic backscattering from tissues. Ultrasonics, Ferroelectrics and Frequency Control, 47(3), 727-736.

[10] Wagner, R.F., Smith, S.W., Sandrik, J.M. and Lopez, H. (1983) Statistics of speckle in ultrasound B-scans. IEEE Transactions on Sonics and Ultrasonics, 30(3), 156-163.

[11] Ghofrani, S., Jahed-Motlagh, M.R. and Ayatollahi, A. (2001) An adaptive speckle suppression filter based on Nakagami distribution. Proceeding of IEEE International Conference on Trends in Communications, Bratislava, 1, 84-87.

[12] Gonzalez, R.C. and Woods, R.E. (2002) Digital image processing. 2nd Edition, Publishing House of Electronics Industry, Beijing, 523-527.

[13] Topiwala, P.N., Ed., (2007) Wavelet image and video compression. The Springer International Series in Engineering and Computer Science, Cambridge.

[14] Vetterli, M. H. (1992) Wavelets and filter banks: Theory and design. IEEE Transactions on Signal Processing, 40 (9), 2207-2232.

[15] Zhenzhu, Y., Yong, Y., Zhenxi, C. and Kongyang, Z. (2009) Study of the de-noising method based on wavelet and fractal. Second International Workshop on Computer Science and Engineering, WCSE'09, 1, 15-19.

[16] Guo, F., Derong, T.L. and Can, Z. (2005) A new compression algorithm for medical images using wavelet transform. IEEE Conferences on Networking, Sensing and Control, Tucson.

[17] Gupta, P.K. and Kanhirodan, R. (2006) Wavelet transform based error concealment approach for image denoising. 1st IEEE Conference on Industrial Electronics and Applications, Singapore.

[18] Xiaojuan, L., Guangshu, H. and Shangkai, G. (1999) Design and implementation of a novel compression method in a tele-ultrasound system. IEEE Transactions on Information Technology in Biomedicine, 3(3), pp. 205-213. 